\title{
Paternité des articles et intérêts concurrents : une analyse des recommandations aux auteurs des journaux traitant de pratique pharmaceutique
}

\author{
Ève Courbon, Cynthia Tanguay, Denis Lebel et Jean-François Bussières
}

\section{RÉSUMÉ}

Contexte : La présence d'auteurs honorifiques et fantômes ainsi que les intérêts concurrents représentent des difficultés bien documentées, liées à la publication d'articles scientifiques. Il existe des lignes directrices encadrant la rédaction et la publication de manuscrits scientifiques, notamment celles de l'International Committee of Medical Journal Editors (ICMJE).

Objectifs : Lobjectif principal de cette étude descriptive et transversale visait à recenser les instructions portant sur la paternité des articles et les intérêts concurrents provenant des recommandations aux auteurs des journaux traitant de pratique pharmaceutique. L'objectif secondaire visait à déterminer des mesures correctrices pour une paternité des articles plus transparente.

Méthode : La recherche a débuté par l'identification des journaux traitant de pratique pharmaceutique. La consultation des instructions aux auteurs des journaux a permis ensuite de recenser les recommandations destinées à éviter les problèmes de paternité des articles et d'intérêts concurrents. Finalement, les membres de l'équipe de recherche se sont consultés afin de définir des mesures correctrices possibles à l'intention des chercheurs.

Résultats : Des 232 journaux traitant de pharmacie, 33 ont été définis comme traitant de pratique pharmaceutique. Un total de 24 (73\%) journaux mentionnaient suivre la politique de l'ICMJE, 14 (42\%) demandaient aux auteurs de remplir un formulaire de déclaration d'intérêts concurrents au moment de la soumission de l'article, 17 (52\%) présentaient une définition de la qualité d'auteur et $5(15 \%)$ demandaient de détailler les contributions de chaque auteur. Une grille de 40 critères a été élaborée pour définir l'attribution du statut d'auteur.

Conclusion : Moins de la moitié des journaux demandait aux auteurs de transmettre un formulaire de déclaration des intérêts concurrents au moment de la soumission d'un article et seulement la moitié des journaux avait donné une définition de la qualité d'auteur. La publication scientifique de travaux sur les pratiques pharmaceutiques n'est pas à l'abri des manques de transparence liés à la publication. L'utilisation d'une grille décrivant la contribution de chaque auteur et la publication en ligne des travaux peuvent contribuer à limiter ces risques.

Mots clés : publication scientifique, pratique pharmaceutique, auteur, intérêts concurrents

\begin{abstract}
Background: Honorary and ghost authorship, as well as competing interests, are well documented concerns related to the publication of scientific articles. Guidelines for writing and publishing scientific manuscripts are available, including those of the International Committee of Medical Journal Editors (ICMJE).

Objectives: The primary objective of this descriptive cross-sectional study was to identify, in the instructions for authors of pharmacy practice journals, guidance on authorship and competing interests. The secondary objective was to suggest suitable corrective measures for more transparent authorship.
\end{abstract}

Methods: The first step of the project was to identify journals in the area of pharmacy practice. The instructions for authors of each journal were then reviewed to determine recommendations for avoiding problems related to authorship and competing interests. Finally, the members of the research team formulated potential corrective measures for researchers.

Results: Of 232 pharmacy journals identified, 33 were deemed to focus on pharmacy practice. A total of $24(73 \%)$ of these journals mentioned that they followed ICMJE policies, 14 (42\%) asked authors to complete a competing interests disclosure form at the time of submission, 17 (52\%) had a formal definition of authorship, and 5 (15\%) asked for details of each author's contribution. A list of 40 criteria was developed to define authorship status.

Conclusion: Fewer than half of the journals asked authors to provide a competing interests disclosure form upon submission of an article, and only half had a formal definition of authorship. The scientific publication of papers relevant to pharmacy practice is not free from issues related to publication transparency. Publishing articles online and using a checklist to detail each author's contribution may help to limit the associated risks.

Keywords: scientific publication, pharmacy practice, author, competing interests

[Publisher's translation] 


\section{INTRODUCTION}

$\mathrm{C}$ réée en 1946, MEDLINE est la source principale de références de PubMed et est alimentée par plus de 5600 journaux indexés 1 . Quant au moteur de recherche PubMed, il existe depuis 1996 et comporte maintenant plus de 22 millions de références ${ }^{2}$. Environ 20 à $25 \%$ des soumissions annuelles de journaux pour indexation à MEDLINE sont retenues par le Literature Selection Technical Review Committee ${ }^{3}$. Le nombre de publications scientifiques est en croissance; par exemple, 23 articles indexés étaient liés au terme MeSH pharmacy en 1945 contre 780 articles en 2012.

Une des difficultés que rencontre la publication d'articles concerne leur paternité, notamment le fait que les critères justifiant le titre d'auteur ne soient pas largement acceptés par la communauté scientifique ${ }^{4-6}$. De plus, l'ordre des auteurs et leur nombre font l'objet de controverses, particulièrement dans le cas d'études multicentriques où le nombre de contributeurs est élevé. Un auteur fantôme est un auteur ayant contribué à la publication d'un article sans y être nommé, et un auteur honorifique est un auteur nommé dans la publication sans y avoir significativement contribué. Selon les études, le pourcentage des auteurs fantômes varie de 18 à 39 \%, et celui des auteurs honorifiques varie entre 1 et $11 \%{ }^{7-12}$. Bien que ces types d'auteurs aient été majoritairement identifiés dans la littérature médicale, le phénomène est plus vaste que cela. Dotson et coll. ${ }^{13}$ ont évalué les caractéristiques des auteurs et des articles publiés dans trois journaux pharmaceutiques (American Journal of Health-System Pharmacy, Annals of Pharmacotherapy et Pharmacotherapy) sur une période de 20 ans, soit en 1989, 1999 et 2009. Leur analyse a montré une augmentation du nombre moyen d'auteurs de 2,5 à 3,6. Les auteurs de l'étude ont conclu que de nombreux facteurs ont contribué à cet accroissement, notamment la pression exercée sur les auteurs pour qu'ils publient, la complexité accrue entourant l'élaboration des protocoles de recherche et l'éthique des auteurs $^{13}$. Dans un sondage réalisé par Dotson et Slaughter ${ }^{12}$ (taux de réponse de $24,9 \%$ ou 114/457) auprès des auteurs d'articles publiés en 2009 dans trois journaux pharmaceutiques, les chercheurs ont pu déterminer un taux de $14 \%$ d'auteurs honorifiques et de $1 \%$ d'auteurs fantômes.

De plus en plus d'essais cliniques publiés sont financés entre autres par l'industrie pharmaceutique, et de plus en plus d'auteurs rapportent des affiliations avec l'industrie ${ }^{13,14}$. Kesselheim et coll. ${ }^{15}$ ont identifié des médecins et des scientifiques ayant prétendument eu des relations financières avec l'industrie pour faire la promotion de médicaments utilisés pour des indications qui ne sont pas prévues par la monographie. Dans les articles publiés par ces mêmes médecins et scientifiques, seulement un auteur sur sept avait déclaré adéquatement les intérêts concurrents (ou conflits d'intérêts) le concernant. Néanmoins, Aneja et coll. ${ }^{16}$ ont observé que les articles pour lesquels des intérêts concurrents étaient déclarés par les auteurs ne rapportaient pas de résultats plus positifs que les autres articles.

Afin de réduire les risques de manque de transparence, des recommandations encadrant la rédaction et la publication de manuscrits scientifiques ont été élaborées par plusieurs organismes. Par exemple, l'International Committee of Medical Journal Editors (ICMJE) produit des recommandations reconnues par de nombreux journaux. Les exigences uniformes pour les manuscrits nommées (" Uniform requirements for manuscripts " [URM]) ont été mises à jour en août 2013 pour devenir les « Recommendations for the conduct, reporting, editing, and publication of scholarly work in medical journals ". Ces recommandations comportent des instructions relatives à la mise en forme et au contenu des soumissions de manuscrits, mais également à la définition de la qualité d'auteur et à la déclaration des intérêts concurrents ${ }^{17}$. Depuis cette mise à jour, l'ICMJE définit la notion d'auteur par l'association des quatre conditions suivantes : 1) contribution substantielle à la conception et à la méthodologie, à l'acquisition des données ou à l'analyse et à l'interprétation des données; 2) écriture ou révision critique d'une part importante du contenu intellectuel de l'article; 3) approbation finale de la version de l'article avant sa publication; 4) prise de la responsabilité de tous les aspects du travail, dont l'exactitude et l'intégrité de l'ensemble du travail.

Lobjectif principal de cette étude descriptive et transversale était de recenser les instructions portant sur la paternité des articles et les intérêts concurrentsprovenant des recommandations aux auteurs de journaux traitant de la pratique pharmaceutique. Lobjectif secondaire visait à déterminer des mesures concrètes pouvant être mises en place au sein d'unités de recherche.

\section{MÉTHODE}

\section{Sélection des journaux traitant de pratique pharmaceutique}

Une recherche dans le catalogue du National Library of Medicine a été effectuée avec le mot clé " pharmac " afin d'identifier les journaux portant sur la pharmacie. Les journaux portant sur la pharmacologie, la toxicologie, la pharmacocinétique, la pharmacognosie, la gestion et l'histoire n'ont pas été retenus. En complément, une recherche utilisant le terme " pharmac " a été effectuée à partir de la liste des journaux de PubMed Central (à l'exception de la catégorie Manuscript Journals), du site Internet de l'ICMJE, de Google et de Google Scholar. Ont été exclus les journaux dans les autres langues que le français et l'anglais. Tout journal n'apparaissant pas dans la recherche mais connu des auteurs a été manuellement ajouté à la collecte. 
À partir de cette première liste de journaux portant sur la pharmacie, ceux traitant de pratique pharmaceutique ont été retenus. Il s'agissait de journaux dont les articles pouvaient décrire et évaluer l'activité pharmaceutique ou pour lesquels il existait une section sur la pratique pharmaceutique. Ils ont été sélectionnés par une assistante de recherche (C.T.) et validés par un deuxième membre de l'équipe recherche (E.C.).

\section{Recensement des recommandations aux auteurs}

Seuls les journaux portant sur la pratique pharmaceutique ont été analysés. Ils ont donné lieu à une collecte d'informations spécifiques, telles que le nom du journal, de son éditeur et du pays de provenance de l'éditeur. Par ailleurs, l'indication de l'indexation du journal dans MEDLINE et dans PubMed Central a été prise en compte. De plus, les chercheurs ont répertorié chaque journal présent sur le site Internet de l'ICMJE. Comme l'étude a été réalisée avant la mise à jour des recommandations de l'ICMJE, les journaux faisaient donc référence aux URM. À noter que la présence d'un journal sur le site de l'ICMJE ne signifie pas nécessairement qu'il respecte toutes ses recommandations. À l'inverse, des journaux ne figurant par sur la liste de l'ICMJE peuvent suivre les recommandations du Comité.

Les mentions suivantes ont été recueillies à partir des instructions aux auteurs, qui étaient disponibles sur les sites Internet des journaux : mention de l'adhésion aux recommandations de l'ICMJE (mise en forme, définition de la qualité d'auteur, nomenclature des références, déclaration d'intérêts concurrents, formulaire de consentement), la présence d'un formulaire d'intérêts concurrents à remplir au moment de la soumission (modèle de l'ICMJE ou autre modèle), la présence de la définition de la qualité d'auteur et la demande de détailler les contributions des auteurs. Il faut noter que la seule mention de l'adhésion du journal aux principes de l'ICMJE ne permettait pas de conclure que la définition de la qualité d'auteur était présente dans les instructions aux auteurs. La collecte de données a été effectuée par deux assistantes de recherche (E.C., C.T.).

\section{Analyse}

Léquipe a procédé au calcul des statistiques descriptives.

\section{Mesures concrètes}

Par la suite, les membres de l'équipe de recherche (E.C., C.T., D.L., J.F.B.) se sont consultés afin de déterminer les mesures concrètes pouvant être mises en place par des unités de recherche afin d'améliorer les pratiques de publication scientifique. Il en a résulté une grille des critères de contribution s'appuyant sur les expériences de l'équipe de recherche et tenant compte des recommandations de l'ICMJE.

\section{RÉSULTATS}

\section{Recommandations aux auteurs des journaux de pratique pharmaceutique}

L'équipe a retenu 244 journaux traitant de pharmacie. Après analyse, elle a retiré 12 doublons (p. ex. le Pharmacy World \& Science est devenu l'International Journal of Clinical Pharmacy), ce qui donne un total de 232 journaux traitant de pharmacie ayant été pris en compte. De ces 232 journaux, 103 (44\%) étaient indexés dans MEDLINE, 93 (40\%) dans PubMed Central et 50 (22\%) apparaissaient sur la liste de l'ICMJE.

Des 232 journaux, 33 traitaient de pratique pharmaceutique (tableau 1). Parmi ces derniers, 15 (45\%) étaient indexés dans MEDLINE, 11 (33\%) dans PubMed Central et 14 (42\%) apparaissaient sur la liste de l'ICMJE. Vingt (20) journaux (61\%) étaient indexés soit dans MEDLINE soit dans PubMed Central.

Au total, 24 journaux $(73 \%)$ mentionnaient qu'ils adhéraient à la politique de l'ICMJE. Cette proportion était plus faible pour les journaux indexés uniquement dans MEDLINE (9/15 ou $60 \%$ ) (figure 1). Quatorze (42\%) journaux traitant de pratique pharmaceutique demandaient un formulaire de déclaration d'intérêts concurrents au moment de la soumission; seulement quatre (12\%) journaux utilisaient exactement le même formulaire que celui disponible sur le site Internet de l'ICMJE. Environ la moitié des journaux (17/33 ou $52 \%$ précisaient leur définition de la qualité d'auteur. Cette proportion était plus faible pour les journaux indexés uniquement dans MEDLINE (6/15 ou $40 \%)$. Très peu (5/33 ou $15 \%$ ) de journaux demandaient de détailler la contribution de chacun des auteurs.

\section{Mesures concrètes}

Une grille de 40 critères définissant la contribution scientifique à la production de manuscrits a été développée (tableau 2). À noter que tous les éléments ne sont pas de valeur égale et que le fait de remplir à un seul critère ne garantit pas forcément le statut d'auteur. Tous les collaborateurs devraient satisfaire à au moins un critère de chacune des quatre premières sections pour être reconnus comme auteurs selon les critères de l'ICMJE.

Parmi les autres mesures concrètes préconisées, notons la création d'une page Internet spécifique au groupe de recherche, comprenant la liste des projets de recherche en cours, le titre de chaque projet, le chercheur principal, la date de début du projet et une mention indiquant si les projets sont en cours ou terminés. Une telle liste pourrait être mise à jour périodiquement. Dans le cas où des travaux ne seraient publiés que sous forme d'affiches présentées à des congrès, l'ajout des affiches en format PDF à un site Internet permettrait 

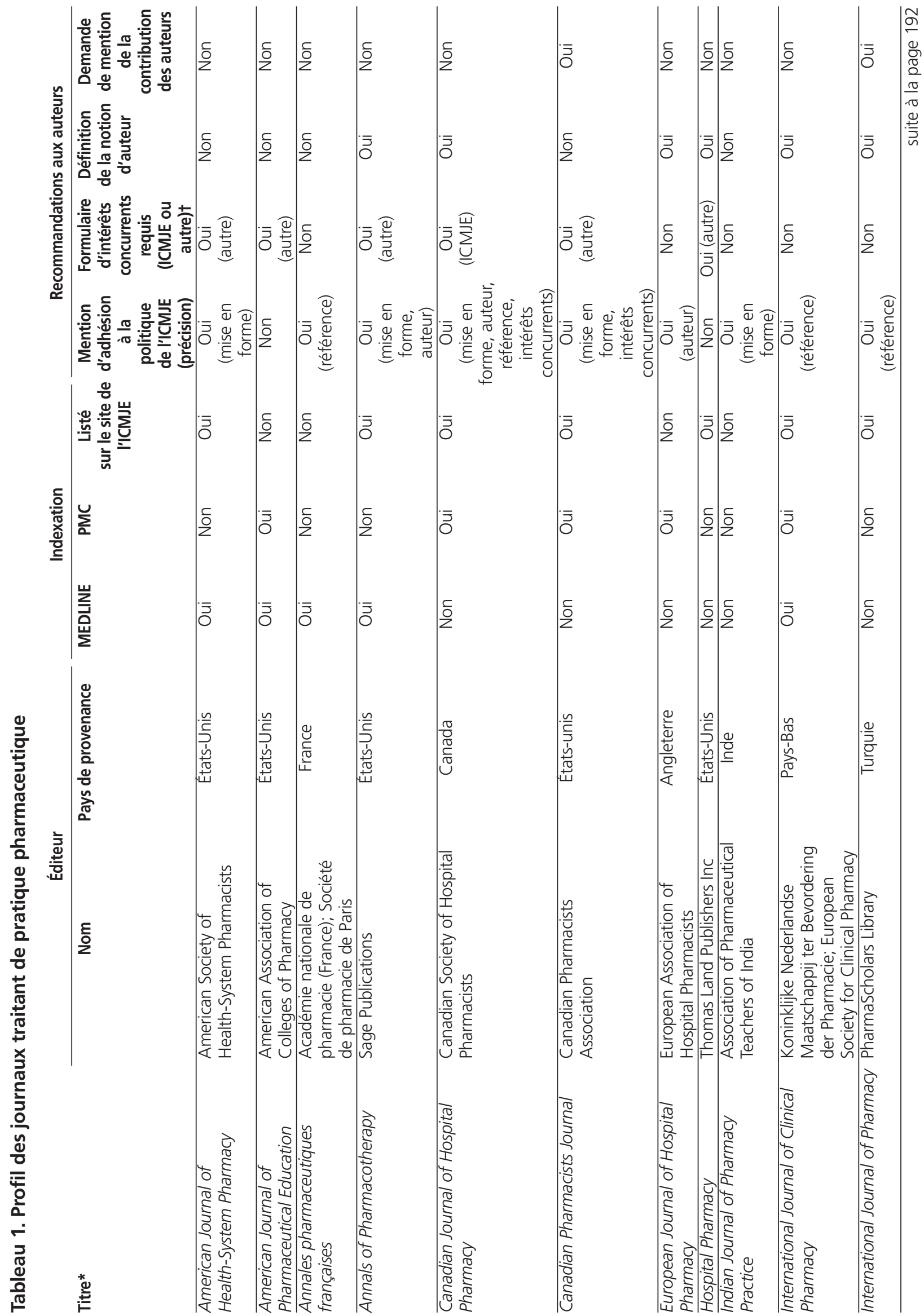

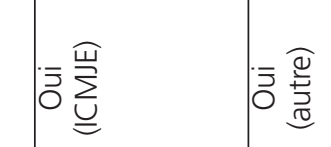

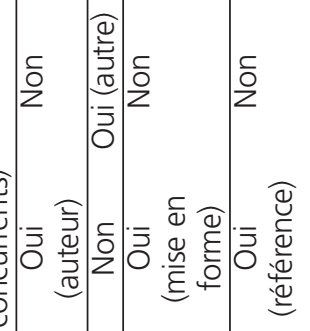

$\frac{\delta}{2}$ ठ̀)

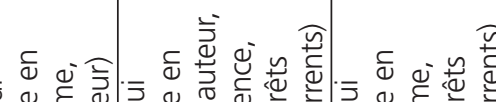

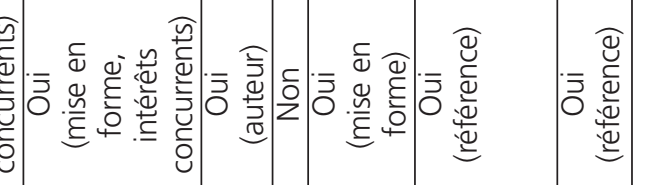

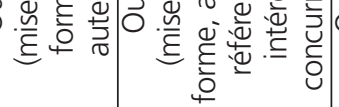

亏े

돌

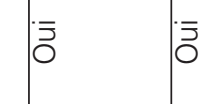

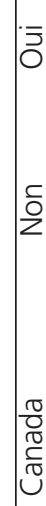



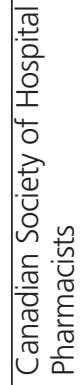

亏ิ

วิ



言
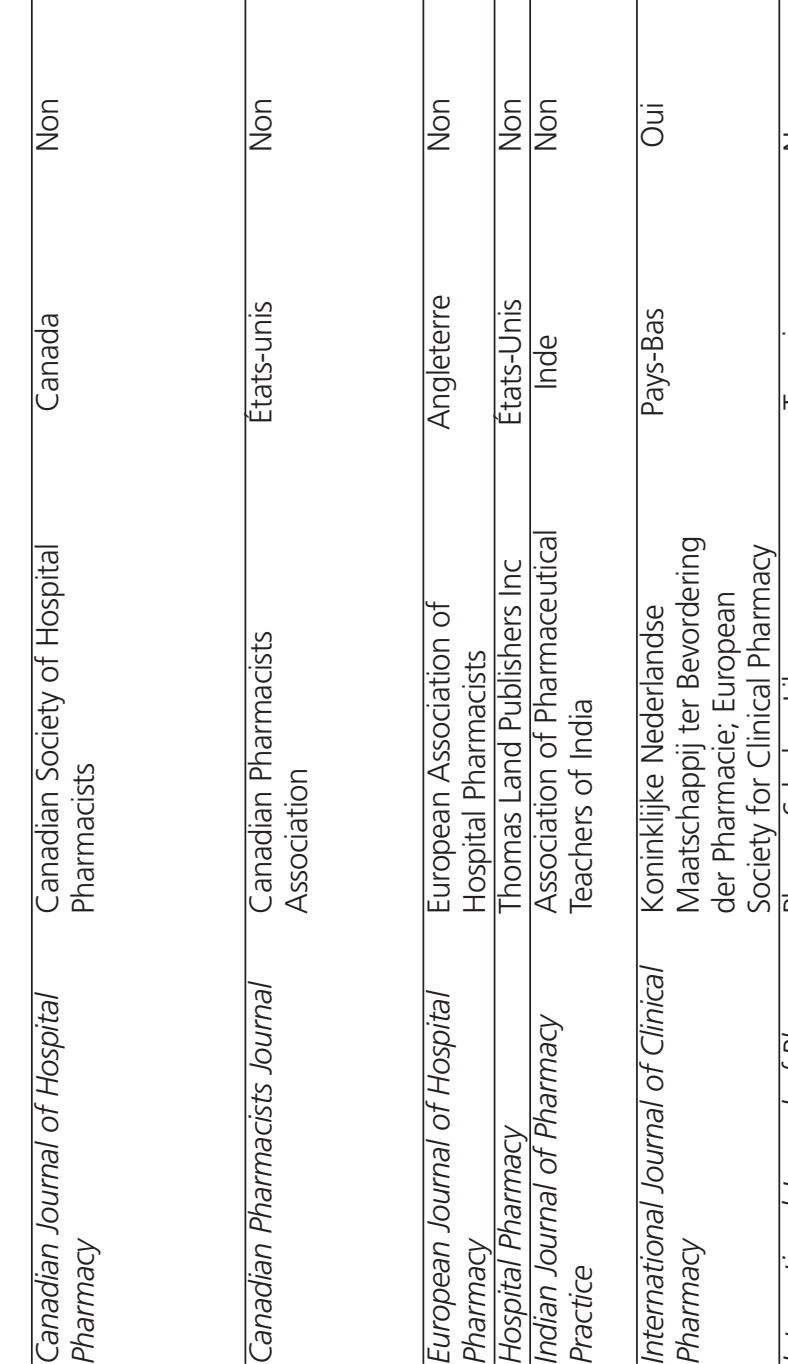

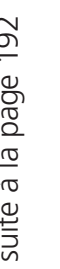



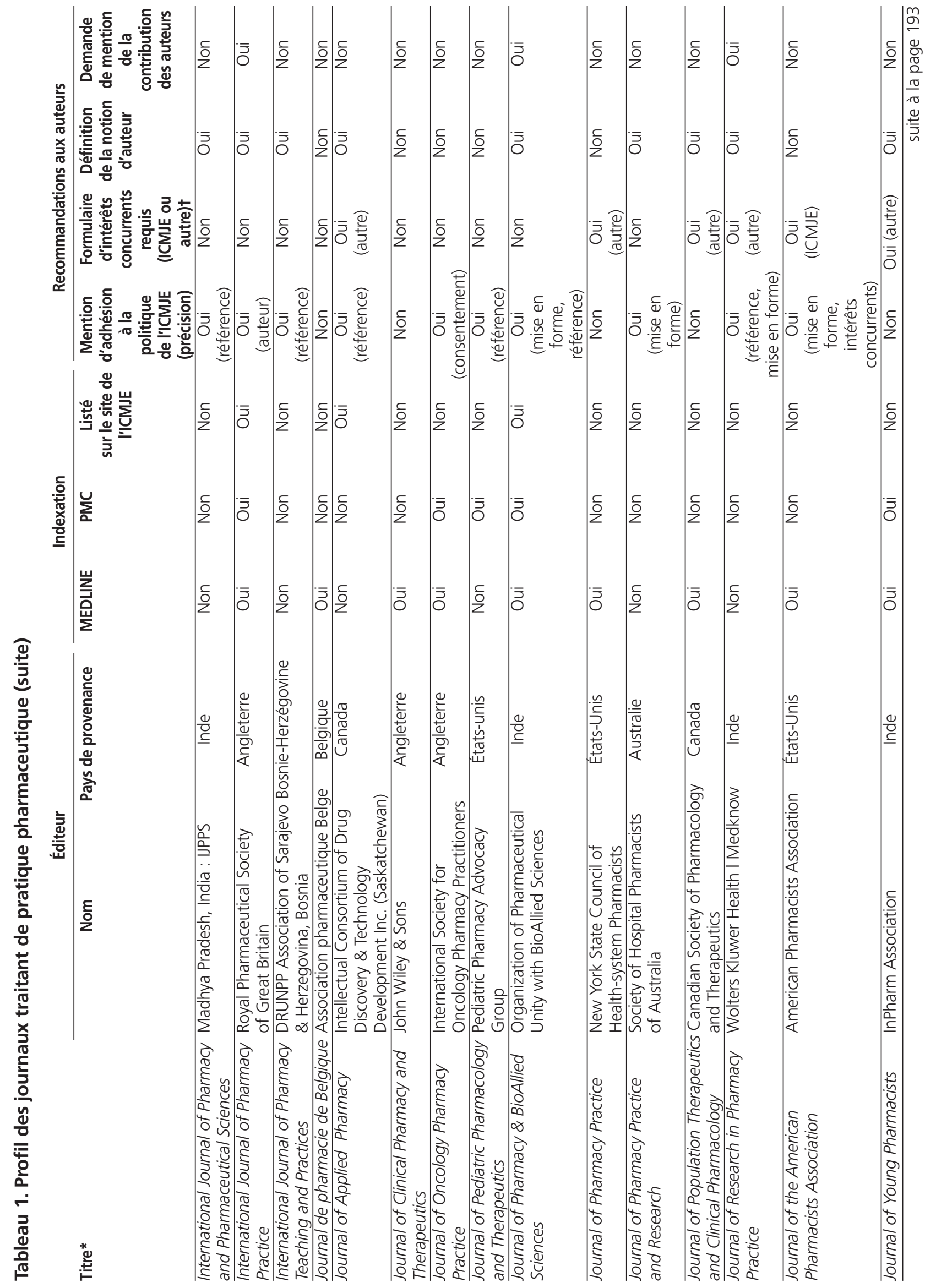
For permission to reprint multiple copies or to order presentation-ready copies for distribution, contact CJHP at cjhpedit@cshp.ca

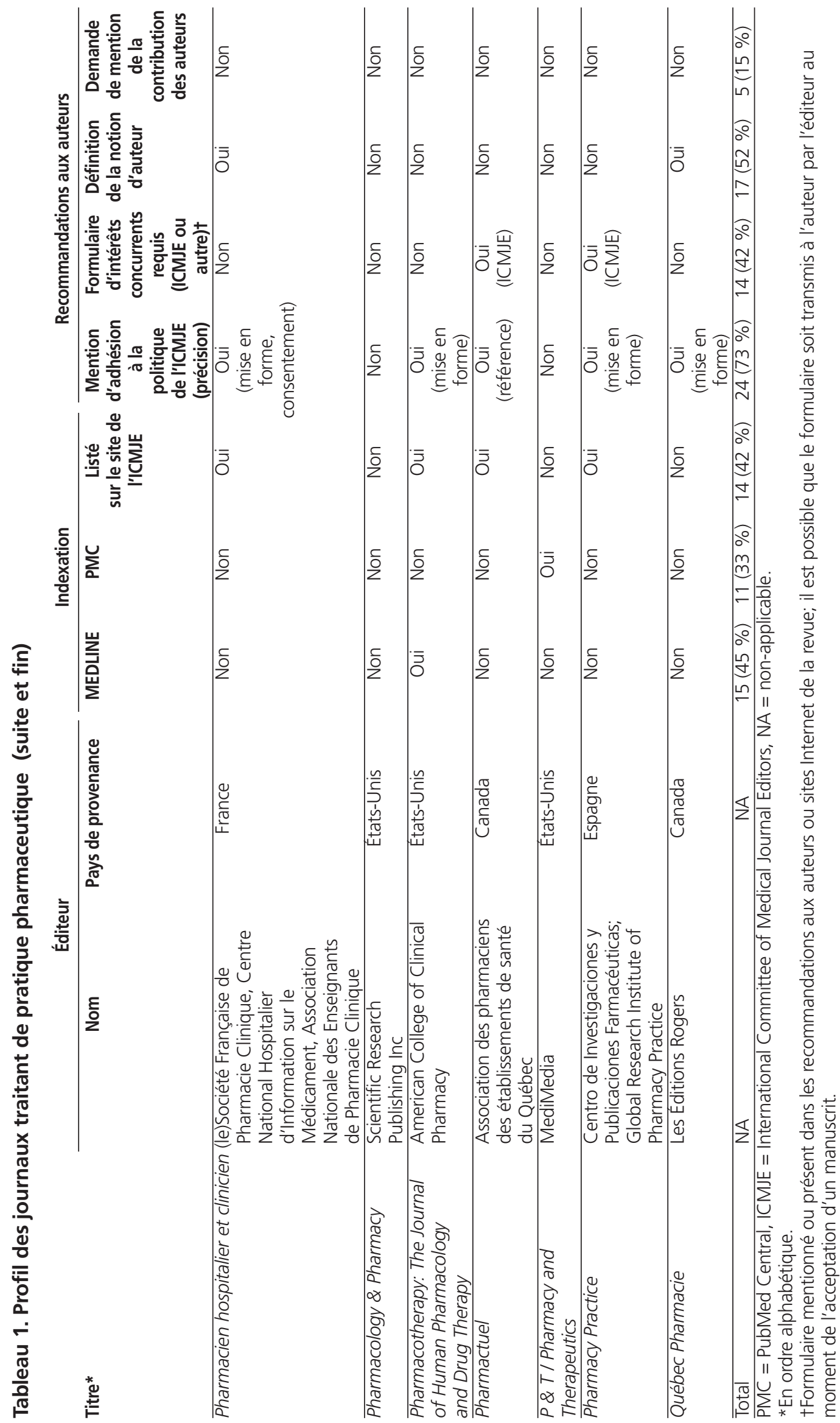




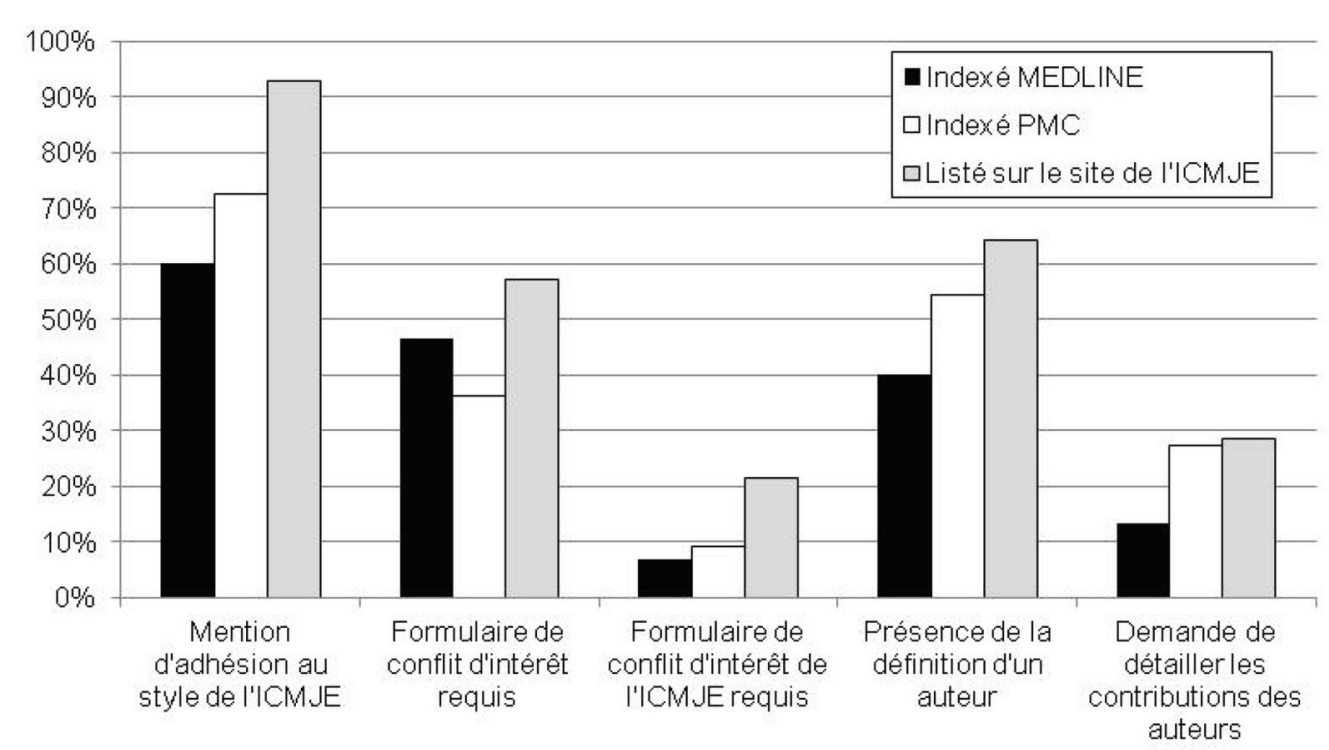

Figure 1. Profil des revues traitant de pratique pharmaceutique selon l'indexation. PMC = PubMed Central, ICMJE = International Committee of Medical Journal Editors.

d'augmenter la visibilité des travaux. Chaque affiche devrait comporter une mention concernant les intérêts concurrents, les sources de financement ainsi que le congrès où la présentation a été faite.

\section{DISCUSSION}

Notre étude descriptive a mis en évidence 33 journaux traitant de pratique pharmaceutique. Plus de la moitié étaient indexés soit dans MEDLINE ou dans PubMed Central. La majorité des journaux adhéraient aux recommandations de l'ICMJE, mais moins de la moitié demandaient de transmettre un formulaire de déclaration des intérêts concurrentsau moment de la soumission.

L'ICMJE n'est pas la seule organisation qui fournisse des recommandations sur les bonnes pratiques de publication scientifique. La World Association of Medical Editors (WAME) a écrit une politique sur les intérêts concurrents en précisant que chaque journal devrait clairement indiquer sa propre définition d'intérêts concurrents et devrait demander aux auteurs d'indiquer par écrit la présence d'intérêts concurrents avant le début du processus de révision ${ }^{18}$. Le Committee on Publication Ethics (COPE) propose des cours en ligne sur l'éthique de la publication, et l'European Association of Science Editors (EASE) propose également des outils de formation en ligne à l'intention des chercheurs ${ }^{19,20}$.

Il est surprenant de constater qu'il n'existe pas encore de consensus sur la définition de la notion d'auteur. Dans notre étude, seulement la moitié des journaux traitant de pratique pharmaceutique avaient précisé la notion d'auteur dans les recommandations aux auteurs, et très peu de journaux ont demandé de détailler les contributions de chacun des auteurs. Étonnamment, les journaux indexés dans MEDLINE ne décrivaient pas plus fréquemment la qualité d'auteur que les journaux non indexés dans MEDLINE.

Les critères de l'ICMJE ont été choisis comme cadre d'élaboration de la grille de contribution. D'autres définitions de la notion d'auteur existent, par exemple l'équipe éditoriale de la revue Neurology demande qu'un auteur ait contribué significativement à au moins un des éléments suivants : contribution intellectuelle substantielle soit à la méthodologie, soit à l'analyse et à l'interprétation des données, soit à l'écriture, soit à la révision critique du contenu du manuscrit ${ }^{21}$. Ainsi, contrairement à l'ICMJE, il n'est pas nécessaire qu'un auteur dans Neurology ait participé à l'analyse des données ainsi qu’à l'écriture du manuscrit. Dans le domaine pharmaceutique, une réflexion semblable s'impose, et les journaux devraient adopter un standard uniforme.

La grille développée par notre équipe (tableau 2) peut être utilisée dès la conception de nouveaux projets de recherche en pratique pharmaceutique et tout au long des travaux, même si la déclaration de contribution des auteurs n'est pas exigée lors de la soumission des manuscrits. Cet outil présente l'avantage de conserver une trace des contributions de chacun, de faciliter l'arbitrage des auteurs lors de la rédaction du manuscrit, de constituer un aide-mémoire utile au moment de la soumission et il représente une bonne pratique en recherche.

Peu importe la définition de la qualité d'auteur que préconise le journal, l'évaluation de l'importance de la contribution considérée comme "significative " est laissée à la 
Tableau 2. Grille des éléments de contribution scientifique à la production de manuscrits*

Éléments de contribution scientifique Auteur $\mathbf{X}[\sqrt{ }]$
à la production de manuscrits
Contribution substantielle à la conception
et à la méthodologie, à la collecte de
données, à leur analyse et à leur
interprétation
1. Détermination de la question de recherche
2. Détermination de la méthodologie et
discussion
3. Identification et sollicitation des
collaborateurs
4. Rédaction du protocole
5. Révision critique du protocole
6. Rédaction du plan d'analyse et de
présentation des résultats
7. Revue documentaire
8. Analyse documentaire
9. Rédaction et validation des outils de
collecte de données
10. Recrutement des patients/participants
11. Participation aux rencontres de l'équipe de
recherche, groupe de discussion, etc.
12. Collecte de données (rétrospective ou
prospective, observations)
13. Saisie de données
14. Extraction de données
15. Analyse statistique des données
16. Mise en forme des données et préparation
17. Interprétation des données
18. Encadrement scientifique du travail des
contributeurs

\section{Contribution substantielle à la rédaction et}

à la révision critique du contenu scientifique

19. Rédaction d'une section du manuscrit

20. Rédaction de l'ensemble du manuscrit

21. Révision critique du contenu

22. Révision linguistique du contenu

\section{Lecture et approbation de la version}

soumise du manuscrit

23. Lecture et approbation du manuscrit soumis

24. Réception de la confirmation de la soumission

discrétion des auteurs. Bien que la grille ne permette pas d'attribuer une importance relative à chaque contribution, elle peut servir de guide à cet effet. Flanagin et coll. ont écrit une liste de 17 critères, tels que réaliser la revue de littérature, réviser le manuscrit, faire la collecte de données et réaliser les analyses statistiques, qui à eux seuls ne sont pas suffisants pour l'attribution du titre d'auteur'. Ivaniš et coll. ont réalisé une étude afin d'évaluer comment les auteurs percevaient l'importance accordée aux différentes contributions possibles à la préparation d'un manuscrit. Les auteurs contactés devaient coter leur participation selon une échelle de Likert à 5 points
Prendre la responsabilité de tous les aspects du travail et assurer l'exactitude et l'intégrité de l'ensemble du travail

25. Consultation de l'ensemble des données afin d'en confirmer l'intégrité et la qualité

26. Capacité de prendre la responsabilité publique du contenu

27. Identification du coauteur responsable de chacun des aspects du travail

Autres contributions potentielles

28. Vérification de l'originalité du manuscrit: que le même contenu n'a pas été publié ailleurs ou autrement

29. Vérification du statut d'auteur : que tous les auteurs le méritent

30. Vérification des contributeurs ne satisfaisant pas à la définition d'auteur : qu'ils sont nommés dans les remerciements et qu'ils ont donné leur accord à cette mention

31. Suivi des communications avec l'éditeur

32. Suivi aux collaborateurs

33. Préparation d'une demande de subvention

34. Aide technique avec l'équipement, les laboratoires

35. Rédaction d'un résumé pour soumission à un congrès

36. Préparation d'une affiche pour présentation à un congrès

37. Préparation d'une communication orale pour présentation à un congrès

38. Présentation à un congrès

39. Révision du manuscrit selon les commentaires de l'éditeur

40. Autre (préciser)

*Pour obtenir le statut d'auteur, les collaborateurs devraient satisfaire à au moins un critère des quatre premières sections. Chaque critère pris isolément n'est pas suffisant pour que soit attribué le statut d'auteur.

(de 0 pour une participation nulle à 4 pour une participation entière). ${ }^{22}$ Les critères de l'ICMJE ont été divisés en 11 catégories de contribution. Ivaniš et coll. ont considéré toute participation supérieure à 2 comme substantielle. De tels exercices réalisés après la publication d'un travail de recherche sont intéressants du fait qu'ils permettent le repérage des enjeux liés à la paternité des articles. Alors que le nombre de publications réalisées par les chercheurs provenant de facultés de pharmacie augmente d'année en année ${ }^{23}$, chaque chercheur devrait être encouragé à dresser la liste de ses contributions dès la conception d'un projet afin de limiter en partie les difficultés liées à l'attribution de la paternité d'un article.

Cette étude descriptive comporte néanmoins des limites. Il est possible que des journaux pertinents pour notre étude aient été omis. Par exemple, nous n'avons pas consulté les journaux indexés dans la banque de données Embase. Lanalyse était limitée aux recommandations aux auteurs disponibles sur les 
sites Internet des journaux. Il est possible que les éditeurs fournissent aux auteurs des instructions et des formulaires supplémentaires plus tard dans le processus de publication des manuscrits. Une recherche complémentaire pourrait donc inclure la vérification des informations recueillies auprès de ces éditeurs. Enfin, notre étude ne permet pas de comparaison avec les revues traitant d'autres sujets que de pratique pharmaceutique.

\section{CONCLUSION}

Notre étude descriptive a pris en compte 33 journaux traitant de pratique pharmaceutique. La majorité des journaux adhérait aux recommandations éditoriales de l'ICMJE, mais moins de la moitié d'entre eux demandaient de transmettre un formulaire de déclaration des intérêts concurrentsau moment de la soumission. Seulement la moitié des journaux avait donné une définition de la notion d'auteur dans ses recommandations aux auteurs, et très peu de journaux ont demandé de détailler les contributions de chacun des auteurs. La publication scientifique de travaux sur les pratiques pharmaceutiques n'est pas à l'abri des pièges liés à la publication. L'utilisation d'une grille décrivant la contribution de chaque auteur et la publication en ligne des travaux de groupes de recherche sur les pratiques pharmaceutiques peut contribuer à limiter ces biais.

\section{Références}

1. Fact sheet MEDLINE®. Bethesda (MD) : US National Library of Medicine; 2013. Publié au www.nlm.nih.gov/pubs/factsheets/medline.html. Consulté le 15 mai 2013.

2. PubMed help: FAQs. Bethesda (MD) : US National Library of Medicine; 2013. Publié au www.ncbi.nlm.nih.gov/books/NBK3827/\#pubmedhelp. FAQs. Consulté le 15 mai 2013.

3. FAQ: journal selection for MEDLINE® indexing at NLM. Bethesda (MD) : US National Library of Medicine; 2013. Publié au www.nlm.nih.gov/ pubs/factsheets/j_sel_faq.html. Consulté le 15 mai 2013.

4. Macrina FL. Teaching authorship and publication practices in the biomedical and life sciences. Sci Eng Ethics. 2011;17(2):341-54.

5. Smith E, Williams-Jones B. Authorship and responsibility in health sciences research: a review of procedures for fairly allocating authorship in multi-author studies. Sci Eng Ethics. 2012;18(2):199-212.

6. Bonita RE, Adams S, Whellan DJ. Reporting of clinical trials: publication, authorship, and trial registration. Heart Fail Clin. 2011;7(4):561-7.

7. Street JM, Rogers WA, Israel M, Braunack-Mayer AJ. Credit where credit is due? Regulation, research integrity and the attribution of authorship in the health sciences. Soc Sci Med. 2010;70(9):1458-65.

8. Shapiro DW, Wenger NS, Shapiro MF. The contributions of authors to multiauthored biomedical research papers. JAMA. 1994;271(6):438-42.

9. Flanagin A, Carey LA, Fontanarosa PB, Philips SG, Pace BP, Lundberg GD, et al. Prevalence of articles with honorary authors and ghosts authors in peerreviewed medical journals. JAMA. 1998;280(3):222-4.

10. Mowatt G, Shirran L, Grimshaw JM, Rennie D, Flanagin A, Yank V, et al. Prevalence of honorary and ghost authorship in Cochrane reviews. JAMA. 2002;287(21):2769-71.

11. Wislar JS, Flanagin A, Fontanarosa PB, Deangelis CD. Honorary and ghost authorship in high impact biomedical journals: a cross sectional survey. $B M J$. 2011;343:d6128.

12. Dotson B, Slaughter RL. Prevalence of articles with honorary and ghost authors in three pharmacy journals. Am J Health Syst Pharm. 2011;68(18): $1730-4$.
13. Dotson B, McManus KP, Zhao JJ, Whittaker P. Authorship and characteristics of articles in pharmacy journals: changes over a 20-year interval. Ann Pharmacother. 2011;45(3):357-63.

14. Buchkowsky SS, Jewesson PJ. Industry sponsorship and authorship of clinical trials over 20 years. Ann Pharmacother. 2004;38(4):579-85.

15. Kesselheim AS, Wang B, Studdert DM, Avorn J. Conflict of interest reporting by authors involved in promotion of off-label drug use: an analysis of journal disclosures. PLoS Med. 2012;9(8):e1001280.

16. Aneja A, Esquitin R, Shah K, Iyengar R, Nisenbaum R, Melo M, et al. Authors' self-declared financial conflicts of interest do not impact the results of major cardiovascular trials. J Am Coll Cardiol. 2013;61(11):1137-43.

17. Recommendations for the conduct, reporting, editing, and publication of scholarly work in medical journals. International Committee of Medical Journal Editors; [révisé aout 2013]. Publié au www.icmje.org/icmjerecommendations.pdf. Consulté le 22 octobre 2013.

18. Conflict of interest in peer-reviewed medical journals. Dallas (TX) : World Association of Medical Editors; 2009. Publié au www.wame.org/conflictof-interest-in-peer-reviewed-medical-journals. Consulté le 11 juillet 2013. (Abonnement requis pour visualiser le contenu.)

19. e-Learning. Committee on Publication Ethics; 2013. Publié au http:// publicationethics.org/resources/elearning. Consulté le 11 juillet 2013.

20. EASE toolkit for authors. Cornwall (UK) : European Association of Science Editors; 2013. Publié au www.ease.org.uk/publications/ease-toolkit-authors. Consulté le 11 juillet 2013.

21. Authorship and contributorship. Dans : Information for authors. Minneapolis (MN) : American Academy of Neurology; 2013. Publié au www.neurology.org/ site/misc/auth2.xhtml. Consulté le 11 juillet 2013.

22. Ivaniš A, Hren D, Marušić M, Marušić A. Less work, less respect: authors' perceived importance of research contributions and their declared contributions to research articles. PLoS One. 2011;6(6):e20206.

23. Chisholm-Burns MA, Spivey C, Martin JR, Wyles C, Ehrman C, Schlesselman LS. A 5-year analysis of peer-reviewed journal article publications of pharmacy practice faculty members. Am J Pharm Educ. 2012;76(7):127.

Ève Courbon est candidate au D. Pharm et assistante de recherche à I'Unité de recherche en pratique pharmaceutique, Centre hospitalier universitaire Sainte-Justine, Montréal, Québec. Elle est aussi interne en pharmacie, Université Paris Sud XI, Paris, France.

Cynthia Tanguay, B. Sc., M. Sc, est coordonnatrice à I'Unité de recherche en pratique pharmaceutique, Centre hospitalier universitaire Sainte-Justine, Montréal, Québec.

Denis Lebel, B. Pharm., M. Sc., FCSHP, est Adjoint, Département de pharmacie et Unité de recherche en pratique pharmaceutique, Centre hospitalier universitaire Sainte-Justine, Montréal, Québec.

Jean-François Bussières, B. Pharm., M. Sc., FCSHP, est Chef, Département de pharmacie et Unité de recherche en pratique pharmaceutique, Centre hospitalier universitaire Sainte-Justine, Montréal, Québec. II est aussi professeur titulaire de clinique, Faculté de pharmacie, Université de Montréal, Montréal, Québec.

Intérêts concurrents : aucun déclaré.

\section{Adresse de correspondance :}

Jean-François Bussières

Département de pharmacie

Centre hospitalier universitaire Sainte-Justine

3175, chemin de la Côte Sainte-Catherine

Montréal QC H3T 1C5

Courriel : jf.bussieres@ssss.gouv.qc.ca 